INTERNATIONAL JOURNAL OF ROBUST AND NONLINEAR CONTROL

Int. J. Robust Nonlinear Control 2009; 19:613-633

Published online 16 June 2008 in Wiley InterScience (www.interscience.wiley.com). DOI: 10.1002/rnc.1336

\title{
Input-to-output stabilization of nonlinear systems via backstepping
}

\author{
D. V. Efimov $1,2, *, \dagger$ and A. L. Fradkov ${ }^{1}$ \\ ${ }^{1}$ Control of Complex Systems Laboratory, Institute of Problem of Mechanical Engineering, \\ Bolshoi av., 61, V.O., St-Petersburg 199178, Russia \\ ${ }^{2}$ Department of Electrical Engineering and Computer Science, B28 Universite de Liege, \\ Sart-Tilman, B-4000 Liege, Belgium
}

\begin{abstract}
SUMMARY
An extension of a backstepping method for the stabilization of nonlinear systems with respect to a set is presented. Robust control laws providing the system with input-to-output stability are proposed. Possibilities of non-strict Lyapunov functions' application are discussed. The differences between a conventional backstepping method and an approach proposed in Kolesnikov (Synergetic Control Theory. Energoatomizdat: Moscow, 1994; 344) are analyzed. Performance of the obtained solutions is demonstrated by computer simulation for pendulum with an actuator example. Copyright (C) 2008 John Wiley \& Sons, Ltd.
\end{abstract}

Received 18 January 2007; Revised 11 February 2008; Accepted 2 April 2008

KEY WORDS: nonlinear control; robust control; partial stabilization; input-to-output stability

\section{INTRODUCTION}

During the last two decades the problem of stabilizing controls design for nonlinear dynamical systems was intensively studied [1] and a number of approaches have been developed. For example, the method of control Lyapunov functions (CLFs) [2-4] gives the necessary and sufficient conditions of continuous controls existence for affine in control nonlinear systems. A feedback linearization approach [5] provides an elegant geometric design tool for a class of nonlinear systems transforming them to linear ones that allows one to apply a wide spectrum of solutions

\footnotetext{
${ }^{*}$ Correspondence to: D. V. Efimov, Control of Complex Systems Laboratory, Institute of Problem of Mechanical Engineering, Bolshoi av., 61, V.O., St-Petersburg 199178, Russia.

†E-mail: efimov@montefiore.ulg.ac.be, efde@mail.rcom.ru

Contract/grant sponsor: Russian Foundation for Basic Research; contract/grant number: 05-01-00869

Contract/grant sponsor: Program of Presidium of Russian Academy of Science; contract/grant number: 22
}

Copyright (C) 2008 John Wiley \& Sons, Ltd. 
available for linear systems. A passification design method is focused on stabilization of a class of nonlinear systems possessing weak minimum phase property [6,7]. There are several methods such as backstepping, nested saturation design or forwarding [1,8-11], which allow one to design stabilizing controls for a class of nonlinear dynamical systems fitting some structural conditions (e.g. well-defined relative degree, minimum phase property, low-triangular model of the plant). There exist robust versions of the above approaches mainly based on input-to-state stability (ISS) theory [12-14].

An important problem of nonlinear control is stabilization with respect to a set (partial stabilization output stabilization) [7,15-20]. Such a problem arises in the areas of oscillations or synchronization control, energy-level stabilization in mechanical systems, maneuvering and in a number of applications in robotics. Robust analogues of stability with respect to a set or with respect to an output were formulated based on input-to-output stability (IOS) concept (an extension of ISS for systems with output) [21-24].

To meet application demands set stabilization design methods have been developed based on CLF [25], feedback linearization [7, 20] and passification [17, 18, 26] approaches. Robust properties of energy-level stabilization control for a pendulum are analyzed in [27]. However, to our best knowledge no results on robust set stabilization by backstepping are available in the literature.

In this paper we attempt to provide such results. We investigate robust properties of proposed control laws based on IOS theory. In Section 2 preliminary results and definitions are summarized. Section 3 contains problem statement and control design results for stabilization with respect to a set. In the second part of Section 3 robust properties of proposed controls are analyzed. The problem of pendulum stabilization is considered in Section 4. Some auxiliary results and proofs are included in Appendices.

\section{PRELIMINARIES}

Let us consider a nonlinear dynamical system

$$
\dot{\mathbf{x}}=\mathbf{f}(\mathbf{x}, \mathbf{u}), \quad \mathbf{y}=\mathbf{h}(\mathbf{x})
$$

where $\mathbf{x} \in R^{n}$ is a state vector; $\mathbf{u} \in R^{m}$ is an input vector; $\mathbf{y} \in R^{p}$ is an output vector; $\mathbf{f}$ and $\mathbf{h}$ are locally Lipschitz continuous vector functions, $\mathbf{h}(0)=0, \mathbf{f}(0,0)=0$. Euclidean norm will be denoted as $|\mathbf{x}|$, and $\|\mathbf{u}\|_{\left[t_{0}, t\right]}$ denotes the $L_{\infty}^{m}$ norm of the input $(\mathbf{u}(t)$ is Lebesgue measurable and locally essentially bounded function $\left.\mathbf{u}: R_{+} \rightarrow R^{m}, R_{+}=\{\tau \in R: \tau \geqslant 0\}\right)$ :

$$
\|\mathbf{u}\|_{\left[t_{0}, T\right]}=\operatorname{ess} \sup \left\{|\mathbf{u}(t)|, t \in\left[t_{0}, T\right]\right\}
$$

if $T=+\infty$ then we will simply express $\|\mathbf{u}\|$. We will denote as $\mathscr{M}_{R^{m}}$ the set of all such Lebesgue measurable inputs $\mathbf{u}$ with a property $\|\mathbf{u}\|<+\infty$ and $\mathscr{M}_{\Omega}$ will be the set of inputs $\mathbf{u}(t) \in \Omega \subset R^{m}$ for almost all $t \geqslant 0$, where $\Omega$ is a compact set. For initial state $\mathbf{x}_{0}$ and input $\mathbf{u} \in \mathscr{M}_{R^{m}}$ let $\mathbf{x}\left(t, \mathbf{x}_{0}, \mathbf{u}\right)$ be the unique maximal solution of (1) (we will use notation $\mathbf{x}(t)$ if all other arguments of solution are clear from the context; $\left.\mathbf{y}\left(t, \mathbf{x}_{0}, \mathbf{u}\right)=\mathbf{h}\left(\mathbf{x}\left(t, \mathbf{x}_{0}, \mathbf{u}\right)\right)\right)$, which is defined on some finite interval $[0, T)$; if solutions are defined for all $t \geqslant 0$ for every initial state $\mathbf{x}_{0}$ and $\mathbf{u} \in \mathscr{M}_{R^{m}}$, then the system is called forward complete. There exists another closely related to forward completeness property 
of system (1): system (1) has unboundedness observability (UO) property, if for each state $\mathbf{x}_{0}$ and input $\mathbf{u} \in \mathscr{M}_{R^{m}}$ such that $T<+\infty$ necessarily

$$
\limsup _{t \rightarrow T}\left|\mathbf{y}\left(t, \mathbf{x}_{0}, \mathbf{u}\right)\right|=+\infty
$$

The necessary and sufficient conditions for forward completeness and UO properties were investigated in [28]. Distance in $R^{n}$ from a given point $\mathbf{x}$ to a set $\mathscr{A}$ is denoted as $|\mathbf{x}|_{\mathscr{A}}=\operatorname{dist}(\mathbf{x}, \mathscr{A})=$ $\inf _{\boldsymbol{\eta} \in \mathscr{A}}|\mathbf{x}-\boldsymbol{\eta}|$. The symbol $D V(\mathbf{x}) \mathbf{F}(\cdot)$ will be stated for directional derivative of a function $V$ with respect to a vector field $\mathbf{F}$ if the function $V$ is differentiable and for the Dini derivative in the direction of $\mathbf{F}$ if the function $V$ is Lipschitz continuous:

$$
D V(\mathbf{x}) \mathbf{F}(\cdot)=\lim _{t \rightarrow 0^{+}} \sup \frac{V(\mathbf{x}+t \mathbf{F}(\cdot))-V(\mathbf{x})}{t}
$$

As usual, the continuous function $\sigma: R_{+} \rightarrow R_{+}$belongs to a class $\mathscr{K}$ if it is strictly increasing and $\sigma(0)=0$; additionally, it belongs to a class $\mathscr{K}_{\infty}$ if it is also radially unbounded; and the continuous function $\beta: R_{+} \times R_{+} \rightarrow R_{+}$is from class $\mathscr{K} \mathscr{L}$, if it is from class $\mathscr{K}$ for the first argument for any fixed second, and it is strictly decreasing to zero by the second argument for any fixed first one.

\subsection{Input-output stability}

Consider system (1) with inputs $\mathbf{u} \in R^{m}$.

Definition 1 (Ingalls and Wang [22] and Sontag and Wang [23])

An UO system (1) is IOS, if there exist $\beta \in \mathscr{K} \mathscr{L}$ and $\gamma \in \mathscr{K}$ such that

$$
\left|\mathbf{y}\left(t, \mathbf{x}_{0}, \mathbf{u}\right)\right| \leqslant \beta\left(\left|\mathbf{x}_{0}\right|, t\right)+\gamma(\|\mathbf{u}\|), \quad t \geqslant 0
$$

holds for all $\mathbf{x}_{0} \in R^{n}$ and $\mathbf{u} \in \mathscr{M}_{R^{m}}$.

Definition 2 (Ingalls and Wang [22])

For system (1), a smooth function $V$ and a function $\lambda: R^{n} \rightarrow R_{+}$are called, respectively, an IOS-Lyapunov function and an auxiliary modulus if there exist $\alpha_{1}, \alpha_{2} \in \mathscr{K}_{\infty}$ such that expression

$$
\alpha_{1}(|\mathbf{h}(\mathbf{x})|) \leqslant V(\mathbf{x}) \leqslant \alpha_{2}(|\mathbf{x}|)
$$

holds and there exist $\chi \in \mathscr{K}$ and $\alpha_{3} \in \mathscr{K} \mathscr{L}$ such that for all $\mathbf{x} \in R^{n}$ and $\mathbf{u} \in R^{m}$,

$$
V(\mathbf{x})>\chi(|\mathbf{u}|) \Rightarrow D V(\mathbf{x}) \mathbf{f}(\mathbf{x}, \mathbf{u}) \leqslant-\alpha_{3}(V(\mathbf{x}), \lambda(\mathbf{x}))
$$

and for all $\mathbf{x}_{0} \in R^{n}$ and all $\mathbf{u} \in \mathscr{M}_{R^{m}}$ there exists some $\delta \in \mathscr{K}$ such that for any $T \geqslant 0$,

$$
V\left(\mathbf{x}\left(t, \mathbf{x}_{0}, \mathbf{u}\right)\right)>\chi(|\mathbf{u}(t)|), \quad t \in[0, T) \Rightarrow \lambda\left(\mathbf{x}\left(t, \mathbf{x}_{0}, \mathbf{u}\right)\right) \leqslant \max \left\{\delta\left(\left|\mathbf{x}_{0}\right|\right), \delta(\|\mathbf{u}\|)\right\}, \quad t \in[0, T)
$$

In [24] IOS-Lyapunov functions was introduced for bounded input-bounded state (BIBS) system (1), for example, when the following inequality is satisfied for all $\mathbf{x}_{0} \in R^{n}$ and $\mathbf{u} \in \mathscr{M}_{R^{m}}$ for some function $\vartheta \in \mathscr{K}$ :

$$
\left|\mathbf{x}\left(t, \mathbf{x}_{0}, \mathbf{u}\right)\right| \leqslant \max \left\{\vartheta\left(\left|\mathbf{x}_{0}\right|\right), \vartheta(\|\mathbf{u}\|)\right\}, \quad t \geqslant 0
$$


In this way one can use $|\mathbf{x}|$ as an auxiliary modulus $\lambda$ (see Remark 3 in [22]) and inequality (3) can be rewritten as follows:

$$
V(\mathbf{x})>\chi(|\mathbf{u}|) \Rightarrow D V(\mathbf{x}) \mathbf{f}(\mathbf{x}, \mathbf{u}) \leqslant-\alpha_{3}(V(\mathbf{x}),|\mathbf{x}|)
$$

Theorem 1 (Ingalls and Wang [22])

Suppose that system (1) is UO. The following are equivalent for the system:

- it is IOS;

- it admits an IOS-Lyapunov function.

\subsection{Uniform output stability}

Assume that inputs $\mathbf{u}$ take values in compact set $\Omega \subset R^{m}$.

Definition 3 (Ingalls and Wang [22] and Sontag and Wang [24])

A forward complete system (1) with inputs from $\mathscr{M}_{\Omega}$ is uniformly output stable (UOS) if for all $\mathbf{x}_{0} \in R^{n}$ and $\mathbf{u} \in \mathscr{M}_{\Omega}$

$$
\left|\mathbf{y}\left(t, \mathbf{x}_{0}, \mathbf{u}\right)\right| \leqslant \beta\left(\left|\mathbf{x}_{0}\right|, t\right), \quad t \geqslant 0, \quad \beta \in \mathscr{K} \mathscr{L}
$$

\section{Definition 4}

For system (1), a smooth function $V$ and a function $\lambda: R^{n} \rightarrow R_{+}$are called, respectively, a UOSLyapunov function and an auxiliary modulus if there exist $\alpha_{1}, \alpha_{2} \in \mathscr{K}_{\infty}$ such that (2) holds and inequality

$$
D V(\mathbf{x}) \mathbf{f}(\mathbf{x}, \mathbf{u}) \leqslant-\alpha_{3}(V(\mathbf{x}), \lambda(\mathbf{x})), \quad \alpha_{3} \in \mathscr{K} \mathscr{L}
$$

is satisfied for all $\mathbf{x}_{0} \in R^{n}$ and all $\mathbf{u} \in \mathscr{M}_{\Omega}$, and $\lambda$ is locally Lipschitz on the set $\{\mathbf{x}: V(\mathbf{x})>0\}$ and $\lambda\left(\mathbf{x}\left(t, \mathbf{x}_{0}, \mathbf{u}\right)\right) \leqslant \lambda\left(\mathbf{x}_{0}\right)$.

The following theorem follows from the results of $[22,24]$.

\section{Theorem 2}

Suppose that system (1) is forward complete and $\mathbf{u} \in \mathscr{M}_{\Omega}$. The following are equivalent for the system:

- it is UOS;

- it admits a UOS-Lyapunov function.

Note that in study [22] UOS property was called output uniform global asymptotic stability. In [25] corresponding CLF formulations are given for tasks of IOS and UOS stabilization; paper [29] presents small gain theorems.

\section{Lemma 1}

Let system (1) with inputs $\mathbf{u} \in \mathscr{M}_{\Omega}$ be UO and there exist a continuously differentiable function $V: R^{n} \rightarrow R_{+}$, which admits (2) and with $\alpha_{0} \in \mathscr{K}$ for all $\mathbf{x} \in R^{n}$ and $\mathbf{u} \in \Omega$

$$
\frac{\partial V}{\partial \mathbf{x}} \mathbf{f}(\mathbf{x}, \mathbf{u}) \leqslant-\alpha_{0}(|\mathbf{y}|)
$$


If a function $\eta(\mathbf{x}, \mathbf{u})=D \mathbf{h}(\mathbf{x}) \mathbf{f}(\mathbf{x}, \mathbf{u})$ is bounded for bounded values of $V(\mathbf{x})$ and $\mathbf{u} \in \mathscr{M}_{\Omega}$, then the system is UOS.

Proofs of the lemma and all theorems are presented in Appendix A. It is said that system (1) is $V$-detectable [17] with respect to continuous function $V: R^{n} \rightarrow R_{+}$on set $\mathscr{X} \subseteq R^{n}$, if for all $\mathbf{x}_{0} \in R^{n}$ and $\mathbf{u}=0$ the following property holds:

$$
\mathbf{x}\left(t, \mathbf{x}_{0}, 0\right) \in \mathscr{X} \quad \forall t \geqslant 0 \Rightarrow \lim _{t \rightarrow+\infty} V\left(\mathbf{x}\left(t, \mathbf{x}_{0}, 0\right)\right)=0
$$

Sufficient conditions for system (1) to be $V$-detectable are presented in [17].

System (1) has a global stability modulo output (GSMO) property if

$$
\left|\mathbf{x}\left(t, \mathbf{x}_{0}, \mathbf{u}\right)\right| \geqslant v\left(\left|\mathbf{y}\left(t, \mathbf{x}_{0}, \mathbf{u}\right)\right|\right), \quad t \in[0, \widetilde{T}) \Rightarrow\left|\mathbf{x}\left(t, \mathbf{x}_{0}, \mathbf{u}\right)\right| \leqslant \max \left\{\mu\left(\left|\mathbf{x}_{0}\right|\right), \mu\left(\|\mathbf{u}\|_{[0, \widetilde{T})}\right)\right\}, \quad t \in[0, \widetilde{T})
$$

here functions $v \in \mathscr{K}_{\infty}, \mu \in \mathscr{K}$ and $\mathbf{x}_{0} \in R^{n}, \mathbf{u} \in \mathscr{M}_{R^{m}}, \widetilde{T}<T$; if $\mathbf{u} \in \mathscr{M}_{\Omega}$, then term $\mu\left(\|\mathbf{u}\|_{[0, \widetilde{T})}\right)$ can be dropped in the last inequality. Boundedness of the output and the GSMO property ensure boundedness of the system state.

\section{MAIN RESULTS}

Let us consider the system

$$
\begin{aligned}
& \dot{\mathbf{x}}=\mathbf{f}\left(\mathbf{x}, \mathbf{z}, \mathbf{d}_{1}\right), \quad \mathbf{y}=\mathbf{h}(\mathbf{x}) \\
& \dot{\mathbf{z}}=\mathbf{u}+\mathbf{d}_{2}
\end{aligned}
$$

where $\mathbf{x} \in R^{n}$ is a state vector of system (5). The system should be stabilized with respect to set $\mathscr{Z}=\{\mathbf{x}: \mathbf{h}(\mathbf{x})=0\}$ defined by the zero level of the output vector $\mathbf{y} \in R^{p} ; \mathbf{z} \in R^{m}$ is a state vector of system (6); $\mathbf{u} \in R^{m}$ is a control vector; $\mathbf{d}_{1} \in R^{q_{1}}, \mathbf{d}_{2} \in R^{q_{2}}$ are vectors of external disturbances, $\mathbf{d}=\left(\mathbf{d}_{1}, \mathbf{d}_{2}\right) \in R^{q}, q=q_{1}+q_{2}$. Functions $\mathbf{h}: R^{n} \rightarrow R^{p}$ and $\mathbf{f}: R^{n+m+q_{1}} \rightarrow R^{n}$ are locally Lipschitz continuous, $\mathbf{f}(0,0,0)=0$. As usual [9] we assume that there exists some continuously differentiable feedback control law $\mathbf{k}: R^{n} \rightarrow R^{m}$ such that system

$$
\dot{\mathbf{x}}=\mathbf{f}\left(\mathbf{x}, \mathbf{k}(\mathbf{x})+\mathbf{e}, \mathbf{d}_{1}\right), \quad \mathbf{y}=\mathbf{h}(\mathbf{x})
$$

possesses UOS or IOS properties with respect to output $\mathbf{y}$ and input $\mathbf{d}_{1}$ for $\mathbf{e}=0$, where variable e corresponds to 'virtual' control realization error $\mathbf{e}=\mathbf{z}-\mathbf{k}(\mathbf{x})$. More precise definition of control $\mathbf{k}$ properties will be described later. Taking into account control $\mathbf{k}$ it is necessary to design the control $\mathbf{u}=\mathbf{U}(\mathbf{x}, \mathbf{z})$, which provides the UOS or IOS property with respect to output $\mathbf{y}$ and input d for the overall system (5), (6). It is the standard backstepping control problem reformulated for input-output stabilization.

Assume that relation

$$
l_{1}(|\mathbf{x}| \mathscr{Z}) \leqslant|\mathbf{h}(\mathbf{x})| \leqslant l_{2}\left(|\mathbf{x}|_{\mathscr{Z}}\right)
$$

is satisfied for some $\imath_{1}, l_{2} \in \mathscr{K}_{\infty}$. Therefore, stability with respect to set $\mathscr{Z}$ in the sense of [21] and UOS with respect to output $\mathbf{y}$ from Definition 3 will be equivalent for us. The above relation means that convergence to zero and boundedness of output function implies the corresponding 
convergence to zero and boundedness of the distance to set $\mathscr{Z}$ and vice versa. Also we use the term 'stabilization with respect to a set' or 'UOS stabilization' instead of the term 'output stabilization' to avoid confusing since in the control literature term 'output stabilization' is frequently used to denote the task of stabilization with respect to a full state vector using feedback dependent on the available for measurements output vector. Here we assume that systems' state vectors $\mathbf{x}$ and $\mathbf{z}$ are available for measurements.

\subsection{UOS stabilization}

Let $\mathbf{d}(t) \equiv 0, t \geqslant 0$. The following assumptions formulate requirements to 'virtual' control $\mathbf{k}$ in this case.

\section{Assumption 1}

There exist continuously differentiable functions $V: R^{n} \rightarrow R_{+}$and $\mathbf{k}: R^{n} \rightarrow R^{m}$ such that

$$
\alpha_{1}(|\mathbf{h}(\mathbf{x})|) \leqslant V(\mathbf{x}) \leqslant \alpha_{2}(|\mathbf{x}|), \quad \alpha_{1}, \alpha_{2} \in \mathscr{K}_{\infty}
$$

and one of the following properties holds for all $\mathbf{x} \in R^{n}, \mathbf{y} \in R^{p}$ and $\mathbf{e} \in R^{m}$ :

1. System (7) is UO and

$$
D V(\mathbf{x}) \mathbf{f}(\mathbf{x}, \mathbf{k}(\mathbf{x}), 0) \leqslant-\alpha_{3}(V(\mathbf{x})), \quad \alpha_{3} \in \mathscr{K}
$$

2. System (7) is UO and

$$
D V(\mathbf{x}) \mathbf{f}(\mathbf{x}, \mathbf{k}(\mathbf{x}), 0) \leqslant-\alpha_{4}(|\mathbf{y}|), \quad \alpha_{4} \in \mathscr{K}
$$

and

$$
|D \mathbf{h}(\mathbf{x}) \mathbf{f}(\mathbf{x}, \mathbf{k}(\mathbf{x})+\mathbf{e}, 0)| \leqslant \mu_{1}(V(\mathbf{x}))+\mu_{2}(|\mathbf{e}|), \quad \mu_{1}, \mu_{2} \in \mathscr{K}
$$

3. System (7) is GSMO or its solutions are bounded and

$$
D V(\mathbf{x}) \mathbf{f}(\mathbf{x}, \mathbf{k}(\mathbf{x}), 0) \leqslant-\alpha_{5}(|\psi(\mathbf{x})|), \quad \alpha_{5} \in \mathscr{K}
$$

and system (7) is $V$-detectable into set $\mathscr{X}=\{\mathbf{x}: \psi(\mathbf{x})=0\}, \psi: R^{n} \rightarrow R^{l}$ is a locally Lipschitz continuous function.

\section{Assumption 2}

There exist continuously differentiable functions $V: R^{n} \rightarrow R_{+}$and $\mathbf{k}: R^{n} \rightarrow R^{m}$ such that (8) is satisfied and one of the following properties holds for all $\mathbf{x} \in R^{n}$ and $\mathbf{e} \in R^{m}$ :

1. System (7) is UO and for $\alpha_{6} \in \mathscr{K}_{\infty}$ and $\sigma_{1} \in \mathscr{K}$

$$
D V(\mathbf{x}) \mathbf{f}(\mathbf{x}, \mathbf{k}(\mathbf{x})+\mathbf{e}, 0) \leqslant-\alpha_{6}(V(\mathbf{x}))+\sigma_{1}(|\mathbf{e}|)
$$

2. System (7) is GSMO or its solutions are bounded and

$$
D V(\mathbf{x}) \mathbf{f}(\mathbf{x}, \mathbf{k}(\mathbf{x})+\mathbf{e}, 0) \leqslant-\alpha_{7}(|\psi(\mathbf{x})|)+\sigma_{2}(|\mathbf{e}|), \quad \alpha_{7} \in \mathscr{K}_{\infty}, \quad \sigma_{2} \in \mathscr{K}
$$

and system (7) is $V$-detectable into set $\mathscr{X}, \psi: R^{n} \rightarrow R^{l}$ is a locally Lipschitz continuous function. 
Let us discuss these assumptions. The difference between them consists in requirements posed on robust properties of control $\mathbf{k}$. In the first assumption, the control provides only the UOS property or its analogues for system (7), whereas the second assumption establishes IOS-like property for system (7) with respect to output $\mathbf{y}$ and input $\mathbf{e}$. If one would require fulfillment of more stronger condition

$$
\alpha_{1}(|\mathbf{h}(\mathbf{x})|) \leqslant V(\mathbf{x}) \leqslant \alpha_{2}(|\mathbf{h}(\mathbf{x})|), \quad \alpha_{1}, \alpha_{2} \in \mathscr{K}_{\infty}
$$

instead of (8), then the first two parts of Assumption 1 would be equivalent. Expression (9) means boundedness of $\dot{\mathbf{y}}$ for bounded values of $V$ and $\mathbf{e}$. According to the results of Theorems 1 and 2 the first parts of Assumptions 1 and 2 imply UOS and IOS properties for system (7) with input e. In this case function $V$ also satisfies (2) from (8) and it is the UOS- or IOS-Lyapunov functions, respectively. Hence, it is possible to weaken these assumptions in the sense of (3) or (4) allowing functions from class $\mathscr{K} \mathscr{L}$ present in the right-hand side of the inequalities for $\dot{V}$.

Before we proceed with the control law design let us note that opposite to the classical work [9] the right-hand side of differential equation (5) depends on $\mathbf{z}$ in a nonlinear manner. To handle this problem we introduce the following supposition.

\section{Assumption 3}

There exists a continuous function $\mathbf{r}: R^{n+m} \rightarrow R^{m}$ such that for all $\mathbf{x} \in R^{n}, \mathbf{z}, \mathbf{z}^{\prime} \in R^{m}$ and $\mathbf{d}_{1} \in R^{q_{1}}$

$$
\frac{\partial V}{\partial \mathbf{x}} \mathbf{f}\left(\mathbf{x}, \mathbf{z}, \mathbf{d}_{1}\right)-\frac{\partial V}{\partial \mathbf{x}} \mathbf{f}\left(\mathbf{x}, \mathbf{z}^{\prime}, \mathbf{d}_{1}\right) \leqslant \mathbf{r}\left(\mathbf{x}, \mathbf{z}^{\prime}\right)^{\mathrm{T}}\left(\mathbf{z}-\mathbf{z}^{\prime}\right)
$$

The last assumption is satisfied for a standard case when $\mathbf{f}\left(\mathbf{x}, \mathbf{z}, \mathbf{d}_{1}\right)=\mathbf{f}_{0}\left(\mathbf{x}, \mathbf{d}_{1}\right)+\mathbf{G}_{0}(\mathbf{x}) \mathbf{z}$, where $\mathbf{f}_{0}$ and $\mathbf{G}_{0}$ are locally Lipschitz continuous vector and matrix functions of appropriate dimensions. In this case, $\mathbf{r}(\mathbf{x})=\partial V / \partial \mathbf{x} \mathbf{G}_{0}(\mathbf{x})$. Also the last assumption is valid, for example, if $\mathbf{f}\left(\mathbf{x}, \mathbf{z}, \mathbf{d}_{1}\right)=$ $\mathbf{f}_{0}\left(\mathbf{x}, \mathbf{d}_{1}\right)+\mathbf{g}(\mathbf{x}, \mathbf{z})$, where $\mathbf{g}$ is a continuously differentiable vector function dependent on variable $\mathbf{z}$ in a concave manner, then $\mathbf{r}(\mathbf{x}, \mathbf{z})=\partial V / \partial \mathbf{x} \partial \mathbf{g} / \partial \mathbf{z}$.

\section{Theorem 3}

Let Assumptions 1 and 3 hold. Then system (5), (6) with a control

$$
\mathbf{u}=\frac{\partial \mathbf{k}}{\partial \mathbf{x}} \mathbf{f}(\mathbf{x}, \mathbf{z}, 0)-\mathbf{r}(\mathbf{x}, \mathbf{k}(\mathbf{x}))-\varphi(\mathbf{z}-\mathbf{k}(\mathbf{x}))
$$

possesses the UOS property, where $\varphi: R^{m} \rightarrow R^{m}$ is a continuous function, $\mathbf{z}^{\mathrm{T}} \varphi(\mathbf{z}) \geqslant \kappa(|\mathbf{z}|)$ for all $\mathbf{z} \in R^{m}, \kappa \in \mathscr{K}$.

\section{Remark 1}

Note that the third part of Assumption 1 covers the second one under supposition that for all $\mathbf{x} \in R^{n}$ and $\mathbf{e} \in R^{m}$

$$
D \psi(\mathbf{x}) \mathbf{f}(\mathbf{x}, \mathbf{k}(\mathbf{x})+\mathbf{e}, 0) \leqslant \mu_{1}(V)+\mu_{2}(|\mathbf{e}|)
$$

for some $\mu_{1}, \mu_{2} \in \mathscr{K}$ instead of GSMO or BIBS properties (of course $\psi$ should be at least locally Lipschitz continuous). Applying the main steps of the theorem proof for this case one can substantiate boundedness of variables $V$ and $\mathbf{e}=\mathbf{z}-\mathbf{k}(\mathbf{x})$. Combining this conclusion with the above inequality and using Lemma 1 we obtain UOS with respect to output $(\boldsymbol{\psi}, \mathbf{e})$. Further, bounded variable $V$ has closed, non-empty, compact and invariant $\omega$-limit set, which obviously belongs to 
a set $\mathscr{X}$ that from $V$-detectability property implies UOS for output $\mathbf{y}$. This fact is the reason for excluding from consideration the second part of Assumption 1 in Assumption 2 and further.

\section{Theorem 4}

Let Assumption 2 hold. Then system (5), (6) with a control

$$
\mathbf{u}=\frac{\partial \mathbf{k}}{\partial \mathbf{x}} \mathbf{f}(\mathbf{x}, \mathbf{z}, 0)-\varphi(\mathbf{z}-\mathbf{k}(\mathbf{x}))
$$

possesses the UOS property, where $\varphi: R^{m} \rightarrow R^{m}$ is a continuous function, $\mathbf{z}^{\mathrm{T}} \varphi(\mathbf{z}) \geqslant \kappa(|\mathbf{z}|)$ for all $\mathbf{z} \in R^{m}, \kappa \in \mathscr{K}$ and $\kappa(s) \geqslant \widetilde{\kappa}(s)+\sigma_{i}(s), \widetilde{\kappa} \in \mathscr{K}$, where $i=1,2$ depending on that part of Assumption 2 holds.

Let us compare the results of Theorems 3 and 4 or the control laws (10) and (11). Theorem 3 needs additional Assumption 3, but it starts from UOS control $\mathbf{k}$. Theorem 4 assumes IOS properties for control $\mathbf{k}$ and it does not require any structural properties of function $\mathbf{f}$ stated in Assumption 3 , but in (11) additional restriction on growth of $\varphi$ is needed. Using terminology from paper [30] one can note that control (10) realizes the so-called 'cancellation backstepping', whereas control (11) corresponds to the so-called ' $L_{g} V$-backstepping'. Also control (11) is close to algorithms proposed in book [8] and it is a part of control (10).

\subsection{IOS stabilization}

In this paragraph we will consider the problem of IOS stabilization of system (5), (6) and some other variants of robustification of controls (10) and (11), when closed-loop system possesses integral variants of the IOS property. This new property is introduced in Appendix B and it is called integral ISS (iISS) with respect to a set by analogy with [31,32]. The main result of Appendix B is formulated in Theorem B1 and proven for the case of compact sets. The problem of compact sets stabilization becomes important in energy desired levels stabilization for mechanical systems. Thus, the result of Theorem B1 can be used to prove robustness with respect to integrally bounded disturbances of passification-based controls $[6,7,16-18]$ usually applied in such situations.

\section{Assumption 4}

There exist continuously differentiable functions $V: R^{n} \rightarrow R_{+}$and $\mathbf{k}: R^{n} \rightarrow R^{m}$ such that (8) is satisfied and one of the following properties holds for all $\mathbf{x} \in R^{n}, \mathbf{e} \in R^{m}$ and $\mathbf{d}_{1} \in R^{q_{1}}$ :

1. System (7) is UO and for $\alpha_{8} \in \mathscr{K}_{\infty}$ and $\sigma_{3} \in \mathscr{K}$

$$
D V(\mathbf{x}) \mathbf{f}\left(\mathbf{x}, \mathbf{k}(\mathbf{x}), \mathbf{d}_{1}\right) \leqslant-\alpha_{8}(V(\mathbf{x}))+\sigma_{3}\left(\left|\mathbf{d}_{1}\right|\right)
$$

2. System (7) is UO and for $\alpha_{9} \in \mathscr{K}_{\infty}$ and $\sigma_{4} \in \mathscr{K}$

$$
D V(\mathbf{x}) \mathbf{f}\left(\mathbf{x}, \mathbf{k}(\mathbf{x})+\mathbf{e}, \mathbf{d}_{1}\right) \leqslant-\alpha_{9}(V(\mathbf{x}))+\sigma_{4}(|\mathbf{e}|)+\sigma_{4}\left(\left|\mathbf{d}_{1}\right|\right)
$$

3. Set $\mathscr{Z}$ is compact and

$$
D V(\mathbf{x}) \mathbf{f}\left(\mathbf{x}, \mathbf{k}(\mathbf{x})+\mathbf{e}, \mathbf{d}_{1}\right) \leqslant-\alpha_{10}(|\psi(\mathbf{x})|)+\sigma_{5}(|\mathbf{e}|)+\sigma_{5}\left(\left|\mathbf{d}_{1}\right|\right), \quad \alpha_{10} \in \mathscr{K}_{\infty}, \quad \sigma_{5} \in \mathscr{K}
$$

and system (7) is $V$-detectable into set $\mathscr{X}, \psi: R^{n} \rightarrow R^{l}$ is a locally Lipschitz continuous function. 
This assumption incorporates into itself Assumptions 1 and 2 for controls (10) and (11). The first part deals with the case of control (10), whereas the last two parts are oriented for the analysis of robust properties of control (11). For shortening of consideration we will consider the case of the third part of Assumption 4 only for control (11), due to the proof and formulation for control (10) are similar.

\section{Assumption 5}

There exists a continuous function $b: R^{n+m} \rightarrow R_{+}$such that for all $\mathbf{x} \in R^{n}$ and $\mathbf{d}_{1}, \mathbf{d}_{1}^{\prime} \in R^{q_{1}}$

$$
\left|\frac{\partial \mathbf{k}}{\partial \mathbf{x}} \mathbf{f}\left(\mathbf{x}, \mathbf{z}, \mathbf{d}_{1}\right)-\frac{\partial \mathbf{k}}{\partial \mathbf{x}} \mathbf{f}\left(\mathbf{x}, \mathbf{z}, \mathbf{d}_{1}^{\prime}\right)\right| \leqslant b(\mathbf{x}, \mathbf{z}) \lambda\left(\left|\mathbf{d}_{1}-\mathbf{d}_{1}^{\prime}\right|\right), \quad \lambda \in \mathscr{K}
$$

If disturbance $\mathbf{d}$ is presented, then we should handle nonlinear dependence of function $\mathbf{f}$ in (5) on vector $\mathbf{d}_{1}$. To do so we introduce the last assumption in the same way as it was done in Assumption 3 for vector $\mathbf{z}$.

\section{Theorem 5}

Let the first part of Assumptions 3-5 hold. Then system (5), (6) with a control

$$
\mathbf{u}=\frac{\partial \mathbf{k}}{\partial \mathbf{x}} \mathbf{f}(\mathbf{x}, \mathbf{z}, 0)-\mathbf{r}(\mathbf{x}, \mathbf{k}(\mathbf{x}))-\varphi(\mathbf{z}-\mathbf{k}(\mathbf{x}))-0.5\left(1+b(\mathbf{x}, \mathbf{z})^{2}\right)(\mathbf{z}-\mathbf{k}(\mathbf{x}))
$$

is IOS provided that $\varphi: R^{m} \rightarrow R^{m}$ is a continuous function, $\mathbf{z}^{\mathrm{T}} \varphi(\mathbf{z}) \geqslant \kappa(|\mathbf{z}|),|\varphi(\mathbf{z})| \geqslant \kappa(|\mathbf{z}|)$ for all $\mathbf{z} \in R^{m}, \kappa \in \mathscr{K}_{\infty}$.

Let the second part of Assumptions 4 and 5 hold. Then system (5), (6) with a control

$$
\mathbf{u}=\frac{\partial \mathbf{k}}{\partial \mathbf{x}} \mathbf{f}(\mathbf{x}, \mathbf{z}, 0)-\varphi(\mathbf{z}-\mathbf{k}(\mathbf{x}))-0.5\left(1+b(\mathbf{x}, \mathbf{z})^{2}\right)(\mathbf{z}-\mathbf{k}(\mathbf{x}))
$$

possesses the IOS property, where $\varphi: R^{m} \rightarrow R^{m}$ is a continuous function, $\mathbf{z}^{\mathrm{T}} \varphi(\mathbf{z}) \geqslant \kappa(|\mathbf{z}|)$, $|\varphi(\mathbf{z})| \geqslant \kappa(|\mathbf{z}|)$ for all $\mathbf{z} \in R^{m}, \kappa \in \mathscr{K}_{\infty}, \kappa(s) \geqslant \widetilde{\kappa}(s)+\sigma_{4}(s), \widetilde{\kappa} \in \mathscr{K}_{\infty}$.

Let the third part of Assumptions 4 and 5 hold, additionally for all $\mathbf{x} \in R^{n}, \alpha_{1}, \alpha_{2} \in \mathscr{K}_{\infty}$

$$
\alpha_{1}(|\mathbf{h}(\mathbf{x})|) \leqslant V(\mathbf{x}) \leqslant \alpha_{2}(|\mathbf{h}(\mathbf{x})|)
$$

Then system (5), (6) with control (13) is iISS with respect to set $\mathscr{A}=\{(\mathbf{x}, \mathbf{z}): \mathbf{x} \in \mathscr{Z}, \mathbf{z}=\mathbf{k}(\mathbf{x})\}$ if $\varphi: R^{m} \rightarrow R^{m}$ is a continuous function, $\mathbf{z}^{\mathrm{T}} \varphi(\mathbf{z}) \geqslant \kappa(|\mathbf{z}|),|\varphi(\mathbf{z})| \geqslant \kappa(|\mathbf{z}|)$ for all $\mathbf{z} \in R^{m}, \kappa \in \mathscr{K}$ and $\kappa(s) \geqslant \widetilde{\kappa}(s)+\sigma_{5}(s), \widetilde{\kappa} \in \mathscr{K}$.

Controls (12) and (13) are robust modifications of controls (10) and (11), respectively. The difference between them consists in the presence of additional feedback with respect to error $\mathbf{e}=\mathbf{z}-\mathbf{k}(\mathbf{x})$ with a functional gain $1+b(\mathbf{x}, \mathbf{z})^{2}$. The first two parts of Theorem 5 deal with a generic case of IOS stabilization, whereas the last part is formulated for a compact set $\mathscr{Z}$ and positive definite with respect to this set function $V$. It is oriented on problems of energy levels' stabilization for mechanical systems [16-18]. 


\subsection{Comparison of different versions of backstepping}

The main advance of controls (11) or (13) (designed with accordance to [8]) with respect to controls (10), (12) consists in explicit independence of equations (11), (13) on the Lyapunov function $V$. These controls are solutions of an auxiliary equation $\dot{\mathbf{e}}+\varphi(\mathbf{e})=0, \mathbf{e}=\mathbf{z}-\mathbf{k}(\mathbf{x})$. Of course, the information about function $V$ is desirable for the analysis of properties of a closed-loop system. However, in some cases it is possible to analyze the closed-loop system properties without using a Lyapunov function $V$, which is not precisely known for designer in a common case. In such situations the following corollary can be applied.

\section{Corollary 1}

Let Assumption 5 hold and UO system (7) be IOS with respect to input (e, $\left.\mathbf{d}_{1}\right)$. Then system (5), (6) with control (13) is forward complete, it has a bounded output $\mathbf{y}$ for any $\mathbf{d} \in \mathscr{M}_{R^{q}}$ and output $\mathbf{y}$ converges to zero for the case without disturbances provided that $\varphi: R^{m} \rightarrow R^{m}$ is continuous and for all $\mathbf{z} \in R^{m}$ inequalities $\mathbf{z}^{\mathrm{T}} \varphi(\mathbf{z}) \geqslant \kappa(|\mathbf{z}|),|\varphi(\mathbf{z})| \geqslant \kappa(|\mathbf{z}|), \kappa \in \mathscr{K}_{\infty}$ hold.

Let Assumption 5 hold and system (7) have convergent to zero output $\mathbf{y}$ for zero input $\left(\mathbf{e}, \mathbf{d}_{1}\right)$ and system be BIBS with respect to input $\left(\mathbf{e}, \mathbf{d}_{1}\right)$. Then system (5), (6) with control (13) has a convergent output $\mathbf{y}$ without disturbances $\mathbf{d}$ and BIBS with respect to input $\mathbf{d}$ under the same requirements to function $\varphi$.

\section{Remark 2}

Let us stress that conditions of the second part of Theorem 5 and in the last corollary are the same. The difference is that in Corollary 1 the conditions are formulated without explicit relying on Lyapunov function $V$. Such a possibility is the important advance of approach proposed in [8] with respect to conventional backstepping [9]. From another side the Lyapunov function method is the main tool for testing and investigation of stability properties of nonlinear dynamical systems.

\section{CONTROL OF A PENDULUM WITH ACTUATOR}

Let us consider the following example of system (5), (6):

$$
\begin{aligned}
& \dot{x}_{1}=x_{2}, \quad \dot{x}_{2}=-x_{1}+z \\
& \dot{z}=u+d_{2}
\end{aligned}
$$

where subsystem (14) corresponds to the forced linear pendulum $\left(x_{1} \in R\right.$ is an angle and $x_{2} \in R$ is the velocity), system (15) models some actuator presence on the input of the pendulum; $d=d_{2} \in R$ is external disturbance. It is necessary to provide nonlinear oscillations of system (14) borrowed from solutions of Van der Pol model:

$$
\dot{x}_{1}=x_{2}, \quad \dot{x}_{2}=-x_{1}+\varepsilon\left(1-x_{1}^{2}\right) x_{2}, \quad \varepsilon>0
$$

Thus, it is required to stabilize the set $\mathscr{Z}$ containing the limit cycle of Van der Pol model plus the origin (it is another invariant unstable solution of the model), note that $\mathscr{Z}$ is a compact set. 
For stabilization of system (14) it is possible to use control

$$
\mathbf{k}\left(x_{1}, x_{2}\right)=\varepsilon\left(1-x_{1}^{2}\right) x_{2}
$$

which for the case $e=0$ ensures convergence of all trajectories to set $\mathscr{Z}$ (in this case controlled system (14) becomes identical to Van der Pol model and set $\mathscr{Z}$ contains all invariant solutions of this model). To apply here the result of Corollary 1 (the second part) it is necessary to prove BIBS property controlled by $\mathbf{k}$ system (14) with respect to input $e$. To do so let us consider the Lyapunov function

$$
V(\mathbf{x})=0.5\left(\varepsilon^{-1} x_{2}-2 x_{1}+\frac{1}{3} x_{1}^{3}\right)^{2}+\frac{1}{12} x_{1}^{4}
$$

where time derivative has the form

$$
\dot{V} \leqslant-\frac{x_{2}^{2}}{2 \varepsilon}-\frac{1}{3 \varepsilon} x_{1}^{4}+\frac{4 \varepsilon^{2}+1}{2 \varepsilon^{2}} x_{1}^{2}+\left|\frac{x_{2}}{\varepsilon}-2 x_{1}+\frac{x_{1}^{3}}{3}\right||e|
$$

It is easy to verify that the following relation holds:

$$
|e| \leqslant 0.25 \min \left\{\left|x_{2}\right|, \varepsilon^{-1}\left|x_{1}\right|\right\} \Rightarrow \dot{V} \leqslant-\frac{x_{2}^{2}}{4 \varepsilon}-\frac{1}{4 \varepsilon} x_{1}^{4}+\left(\frac{4 \varepsilon^{2}+\varepsilon+1}{2 \varepsilon^{2}}\right) x_{1}^{2}
$$

that implies BIBS property of the system. Since in this example $d_{1}=0$, then there is no need to check Assumption 5. Substitute $b\left(x_{1}, x_{2}, z\right)=1$, then according to Corollary 1 control

$$
u=\varepsilon\left(1-x_{1}^{2}\right)\left(z-x_{1}\right)-2 \varepsilon x_{2}^{2} x_{1}-K\left[z-\varepsilon x_{2}\left(1-x_{1}^{2}\right)\right]
$$

where $\varphi(\mathbf{z})=K \mathbf{z}, K>0$ provides for system (14), (15) output convergence and BIBS properties. The trajectories of system (14)-(16) for $K=1, \varepsilon=1, d_{2}(t)=0.2 \sin (t)$ are shown in Figure 1 . As it is possible to conclude from Figure 1(a) all trajectories of the system converge to small neighborhood of set $\mathscr{Z}$ in the plane $\left(x_{1}, x_{2}\right)$. Size of the neighborhood is proportional to the amplitude of disturbance $d_{2}$. In Figure 1(b) oscillating behavior of the system in coordinates $\left(x_{1}, x_{2}\right)$ is shown.

The continuous control (16) provides almost global stabilization of the limit cycle for system (14), (15). To ensure continuity of the control we include in a goal set $\mathscr{Z}$ the unstable equilibrium at the origin. That allows us to consider the problem of compact set stabilization applying conventional stability paradigms (not using almost all stability or multi-stability frameworks) and to investigate robustness of the system against disturbances.

\section{CONCLUSION}

This paper presents an extension of backstepping technique for the problem of stabilization with respect to a set or UOS stabilization. Robust version of this result for IOS stabilization is also proposed. Introduced in Appendix B a new iISS property with respect to a set helps to establish robust properties of passification controls in tasks of energy-level stabilization. The performance 

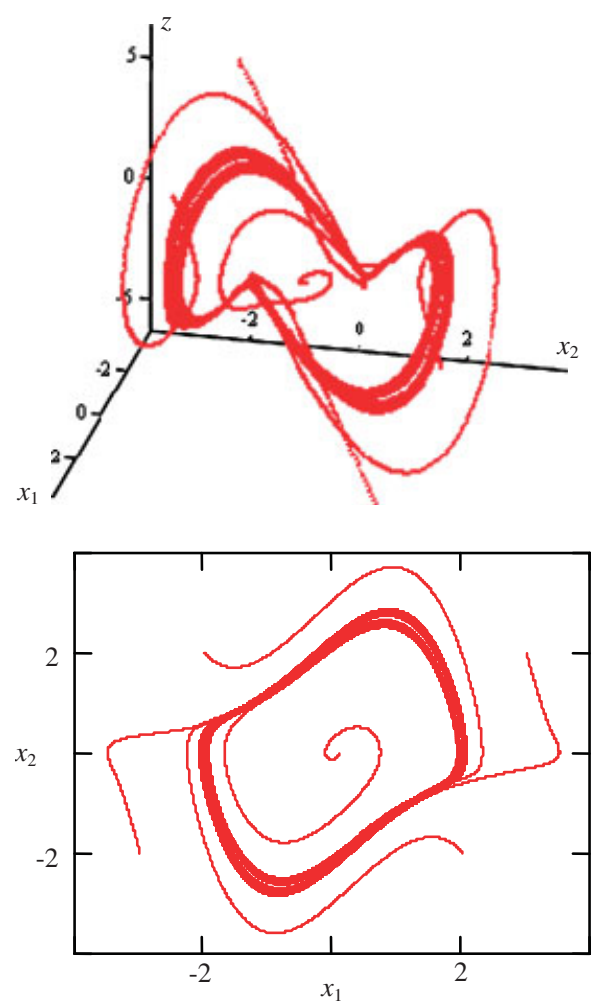

Figure 1. Trajectories of system (14)-(16).

of the proposed solution is demonstrated through analytical design and computer simulation of pendulum stabilization problem in the presence of disturbances and input actuator.

\section{APPENDIX A}

Proof of Lemma 1

First, note that $\dot{V} \leqslant 0$ and from (2) variable $\mathbf{y}$ is bounded and for any $\mathbf{x}_{0} \in R^{n}$ and $\mathbf{u} \in \mathscr{M}_{\Omega}$

$$
\left|\mathbf{y}\left(t, \mathbf{x}_{0}, \mathbf{u}\right)\right| \leqslant \alpha_{1}^{-1} \circ \alpha_{2}\left(\left|\mathbf{x}_{0}\right|\right), \quad t \geqslant 0
$$

From UO property solutions of the system are well defined for all $t \geqslant 0$.

Let $\left|\mathbf{x}_{0}\right| \leqslant \delta, \delta>0$. Then for each $\varepsilon>0$ there exists $T=T(\delta, \varepsilon)>0$ such that $|\mathbf{y}(t)| \leqslant \varepsilon$ for all $t \geqslant T$. Indeed, it follows from the estimate for $\dot{V}$ that for any $\varepsilon>0$ there exists $T_{0}>0$ with $\left|\mathbf{y}\left(T_{0}\right)\right|=\varepsilon / 2$. If it is not the case, then for all $t \geqslant 0$ solution of (1) obeys inequality $\dot{V} \leqslant-\alpha_{0}(\varepsilon / 2)$, that is a contradiction. Suppose that there exists instant of time $T_{1}>T_{0}$, such that $\left|\mathbf{y}\left(T_{1}\right)\right|>\varepsilon$ (if it does not, then claim holds with $T=T_{0}$ ). By the same arguments, there exists instant of time $T_{2}>T_{1}$ such that $\left|\mathbf{y}\left(T_{2}\right)\right|=\varepsilon / 2$. In this way we can construct an increasing sequence of time instants $T_{0}, T_{1}, T_{2}, \ldots$ with $\left|\mathbf{y}\left(T_{2 k}\right)\right|=\varepsilon / 2$ and $\left|\mathbf{y}\left(T_{2 k+1}\right)\right|>\varepsilon, k=0,1,2, \ldots$. Owing to boundedness of 
function $\eta$ this sequence has an accumulation point only at infinity and there exists a $\Delta_{T}=$ $\Delta_{T}(\delta, \varepsilon)>0$ such that $\min _{k \geqslant 0}\left(T_{2 k+1}-T_{2 k}\right) \geqslant \Delta_{T}$. If for some $k \geqslant 0$ there is no $T_{2 k+1}$, then this sequence is finite and claim is true with $T=T_{2 k}$. Suppose that there exist $T_{2 k+1}$ with the abovedescribed property for all $k \geqslant 0$. It means that there exist infinite series of intervals $\left[T_{2 k}^{\prime}, T_{2 k+1}\right)$, $T_{2 k}^{\prime}=\arg \sup _{t \in\left[T_{2 k}, T_{2 k+1}\right)}\{t:|\mathbf{y}(t)|=\varepsilon / 2\}$, which admit property $\dot{V}(t) \leqslant-\alpha_{0}(\varepsilon / 2), t \in\left[T_{2 k}^{\prime}, T_{2 k+1}\right)$. Thus, according to the negative semidefinite property of function $\dot{V}$ inequalities

$$
V\left(T_{2 k+1}\right) \leqslant V\left(T_{2 k}^{\prime}\right) \leqslant V\left(T_{2 k}\right), \quad V\left(T_{2 k+1}\right) \leqslant V\left(T_{2 k}^{\prime}\right)-\Delta_{T}^{\prime} \alpha_{0}(\varepsilon / 2), \quad \min _{k \geqslant 0}\left(T_{2 k+1}-T_{2 k}^{\prime}\right) \geqslant \Delta_{T}^{\prime}
$$

hold for all $k \geqslant 0$. Hence, on each time interval $\left[T_{2 k}, T_{2 k+1}\right.$ ) value of function $V$ decreases at least on $\Delta_{T}^{\prime} \alpha_{0}(\varepsilon / 2)$, but it is a contradiction due to finiteness of $V\left(T_{0}\right) \leqslant \alpha_{2}(\delta)$. Hence, the sequence $T_{0}, T_{1}, T_{2}, \ldots, T_{2 k}$ is finite for some $k \geqslant 0$. Note that the exact value of $k$ depends only on initial conditions, which are characterized by $\delta$ and the value of $\varepsilon$; hence, $T=T(\delta, \varepsilon)$. Existence of the estimate with $\mathscr{K} \mathscr{L}$ function as in Definition 3 can be substantiated as in Proposition 2.5 in [21] and system (1) is UOS.

\section{Proof of Theorem 3}

Let us consider the following Lyapunov function:

$$
U(\mathbf{x}, \mathbf{z})=V(\mathbf{x})+0.5(\mathbf{z}-\mathbf{k}(\mathbf{x}))^{\mathrm{T}}(\mathbf{z}-\mathbf{k}(\mathbf{x}))
$$

where the time derivative for system (5), (6) with Assumption 3 takes the form

$$
\begin{aligned}
\dot{U}= & D V(\mathbf{x}) \mathbf{f}(\mathbf{x}, \mathbf{z}, 0)+(\mathbf{z}-\mathbf{k}(\mathbf{x}))^{\mathrm{T}}(\mathbf{u}-\partial \mathbf{k} / \partial \mathbf{x f}(\mathbf{x}, \mathbf{z}, 0)) \\
\leqslant & D V(\mathbf{x}) \mathbf{f}(\mathbf{x}, \mathbf{z}, 0)-D V(\mathbf{x}) \mathbf{f}(\mathbf{x}, \mathbf{k}(\mathbf{x}), 0)+D V(\mathbf{x}) \mathbf{f}(\mathbf{x}, \mathbf{k}(\mathbf{x}), 0) \\
& +(\mathbf{z}-\mathbf{k}(\mathbf{x}))^{\mathrm{T}}(\mathbf{u}-\partial \mathbf{k} / \partial \mathbf{x f}(\mathbf{x}, \mathbf{z}, 0)) \\
\leqslant & D V(\mathbf{x}) \mathbf{f}(\mathbf{x}, \mathbf{k}(\mathbf{x}), 0) \\
& +\mathbf{r}(\mathbf{x}, \mathbf{k}(\mathbf{x}))^{\mathrm{T}}(\mathbf{z}-\mathbf{k}(\mathbf{x}))+(\mathbf{z}-\mathbf{k}(\mathbf{x}))^{\mathrm{T}}(\mathbf{u}-\partial \mathbf{k} / \partial \mathbf{x f}(\mathbf{x}, \mathbf{z}, 0))
\end{aligned}
$$

Substituting in the above inequality control (10) we obtain

$$
\dot{U} \leqslant D V(\mathbf{x}) \mathbf{f}(\mathbf{x}, \mathbf{k}(\mathbf{x}), 0)-(\mathbf{z}-\mathbf{k}(\mathbf{x}))^{\mathrm{T}} \varphi(\mathbf{z}-\mathbf{k}(\mathbf{x}))
$$

For all parts of Assumption 1 we have property $D V(\mathbf{x}) \mathbf{f}(\mathbf{x}, \mathbf{k}(\mathbf{x}), 0) \leqslant 0$. Hence, $\dot{U} \leqslant 0$ and variables $V$ and $\mathbf{e}=\mathbf{z}-\mathbf{k}(\mathbf{x})$ are bounded that due to UO property of system (7) means forward completeness of the overall system. If the first part of Assumption 1 is satisfied, then

$$
\dot{U} \leqslant-\alpha_{3}(V)-(\mathbf{z}-\mathbf{k}(\mathbf{x}))^{\mathrm{T}} \varphi(\mathbf{z}-\mathbf{k}(\mathbf{x})) \leqslant-\widetilde{\alpha}_{3}(U), \quad \widetilde{\alpha}_{3}(s)=\min \left\{\alpha_{3}(0.5 s), \kappa(\sqrt{s})\right\}
$$

That is equivalent to (4) and $U$ is a UOS Lyapunov function for the system. Let the second part of Assumption 1 hold, then estimate (A2) can be rewritten as follows:

$$
\dot{U} \leqslant-\alpha_{4}(|\mathbf{y}|)-(\mathbf{z}-\mathbf{k}(\mathbf{x}))^{\mathrm{T}} \varphi(\mathbf{z}-\mathbf{k}(\mathbf{x}))
$$


Using (9) and boundedness of $\mathbf{e}$ and $V$ one can substantiate boundedness of $\dot{\mathbf{y}}$. Thus, the UOS property follows from Lemma 1. Finally, let the third part of Assumption 1 be satisfied. Then (A2) has the form

$$
\dot{U} \leqslant-\alpha_{5}(|\psi|)-(\mathbf{z}-\mathbf{k}(\mathbf{x}))^{\mathrm{T}} \varphi(\mathbf{z}-\mathbf{k}(\mathbf{x}))
$$

and from boundedness of $\mathbf{y}, \mathbf{e}$ and GSMO property of system (7) the boundedness of all system trajectories can be obtained. And again the UOS property with respect to extended output $(\boldsymbol{\psi}, \mathbf{e})$ follows from Lemma 1. Additionally, due to boundedness of the system state vector its $\omega$-limit trajectories belong to a set $\mathscr{X}$ that according to $V$-detectability property implies convergence of $\mathbf{y}$ to zero.

\section{Proof of Theorem 4}

Let us consider the Lyapunov function (A1), where the time derivative for system (5), (6), (11) can be rewritten as follows:

$$
\dot{U} \leqslant D V(\mathbf{x}) \mathbf{f}(\mathbf{x}, \mathbf{k}(\mathbf{x}), 0)-\kappa(|\mathbf{z}-\mathbf{k}(\mathbf{x})|)
$$

Assume that the first part of Assumption 2 is satisfied and the feedback function $\varphi$ provides the fulfillment of inequality $\kappa(s) \geqslant \widetilde{\kappa}(s)+\sigma_{1}(s)$. Then (A3) can be rewritten in the form

$$
\dot{U} \leqslant-\alpha_{6}(V)-\widetilde{\kappa}(|\mathbf{z}-\mathbf{k}(\mathbf{x})|) \leqslant-\widetilde{\alpha}_{6}(U), \quad \widetilde{\alpha}_{6}(s)=\min \left\{\alpha_{6}(0.5 s), \widetilde{\kappa}(\sqrt{s})\right\}
$$

that means boundedness of variables $V$ and $\mathbf{e}=\mathbf{z}-\mathbf{k}(\mathbf{x})$. Combining the above facts with the UO property we obtain forward completeness of the system. Here $U$ is the UOS Lyapunov function for the system and the result follows from Theorem 2. Now assume that the second part of Assumption 2 is valid and the feedback function $\varphi$ satisfies inequality $\kappa(s) \geqslant \widetilde{\kappa}(s)+\sigma_{2}(s)$. Estimate (A3) takes the form

$$
\dot{U} \leqslant-\alpha_{7}(|\psi|)-\widetilde{\kappa}(|\mathbf{z}-\mathbf{k}(\mathbf{x})|)
$$

Condition $\dot{U} \leqslant 0$ provides boundedness of variables $V$ and e that with GSMO property is equivalent to boundedness of variable $\mathbf{x}$. Applying Lemma 1 we obtain UOS with respect to variable $(\boldsymbol{\psi}, \mathbf{e})$. Boundedness of the system state vector implies existence of its $\omega$-limit set, which belong to a set $\mathscr{X}$ that according to $V$-detectability property results to convergence of $\mathbf{y}$ to zero.

Proof of Theorem 5

Let us consider the Lyapunov function (A1), where the time derivative for system (5), (6) with Assumption 3 takes the form

$$
\begin{aligned}
\dot{U}= & D V(\mathbf{x}) \mathbf{f}\left(\mathbf{x}, \mathbf{z}, \mathbf{d}_{1}\right)+(\mathbf{z}-\mathbf{k}(\mathbf{x}))^{\mathrm{T}}\left(\mathbf{u}+\mathbf{d}_{2}-\partial \mathbf{k} / \partial \mathbf{x f}\left(\mathbf{x}, \mathbf{z}, \mathbf{d}_{1}\right)\right) \\
\leqslant & D V(\mathbf{x}) \mathbf{f}\left(\mathbf{x}, \mathbf{k}(\mathbf{x}), \mathbf{d}_{1}\right)+\mathbf{r}(\mathbf{x}, \mathbf{k}(\mathbf{x}))^{\mathrm{T}}(\mathbf{z}-\mathbf{k}(\mathbf{x})) \\
& +(\mathbf{z}-\mathbf{k}(\mathbf{x}))^{\mathrm{T}}\left(\mathbf{u}+\mathbf{d}_{2}-\partial \mathbf{k} / \partial \mathbf{x} \mathbf{f}\left(\mathbf{x}, \mathbf{z}, \mathbf{d}_{1}\right)\right)
\end{aligned}
$$


Substituting in the above inequality control (12) and applying Young's inequality $2 a b \leqslant a^{2}+b^{2}$ for $a, b \in R$ with Assumption 5 we obtain

$$
\begin{aligned}
\dot{U} \leqslant & D V(\mathbf{x}) \mathbf{f}\left(\mathbf{x}, \mathbf{k}(\mathbf{x}), \mathbf{d}_{1}\right)-(\mathbf{z}-\mathbf{k}(\mathbf{x}))^{\mathrm{T}} \varphi(\mathbf{z}-\mathbf{k}(\mathbf{x})) \\
& +0.5\left|\mathbf{d}_{2}\right|^{2}-0.5 b(\mathbf{x}, \mathbf{z})^{2}|\mathbf{z}-\mathbf{k}(\mathbf{x})|^{2}+(\mathbf{z}-\mathbf{k}(\mathbf{x}))^{\mathrm{T}} \\
& \times\left(\partial \mathbf{k} / \partial \mathbf{x} \mathbf{f}(\mathbf{x}, \mathbf{z}, 0)-\partial \mathbf{k} / \partial \mathbf{x} \mathbf{f}\left(\mathbf{x}, \mathbf{z}, \mathbf{d}_{1}\right)\right) \\
\leqslant & D V(\mathbf{x}) \mathbf{f}\left(\mathbf{x}, \mathbf{k}(\mathbf{x}), \mathbf{d}_{1}\right)-(\mathbf{z}-\mathbf{k}(\mathbf{x}))^{\mathrm{T}} \varphi(\mathbf{z}-\mathbf{k}(\mathbf{x})) \\
& +0.5\left|\mathbf{d}_{2}\right|^{2}-0.5 b(\mathbf{x}, \mathbf{z})^{2}|\mathbf{z}-\mathbf{k}(\mathbf{x})|^{2}+b(\mathbf{x}, \mathbf{z})|\mathbf{z}-\mathbf{k}(\mathbf{x})| \lambda\left(\left|\mathbf{d}_{1}\right|\right) \\
\leqslant & D V(\mathbf{x}) \mathbf{f}\left(\mathbf{x}, \mathbf{k}(\mathbf{x}), \mathbf{d}_{1}\right)-(\mathbf{z}-\mathbf{k}(\mathbf{x}))^{\mathrm{T}} \varphi(\mathbf{z}-\mathbf{k}(\mathbf{x}))+0.5 \lambda\left(\left|\mathbf{d}_{1}\right|\right)^{2}+0.5\left|\mathbf{d}_{2}\right|^{2}
\end{aligned}
$$

According to the first part of Assumption 4 the last estimate can be rewritten as follows:

$$
\dot{U} \leqslant-\alpha_{8}(V(\mathbf{x}))-(\mathbf{z}-\mathbf{k}(\mathbf{x}))^{\mathrm{T}} \varphi(\mathbf{z}-\mathbf{k}(\mathbf{x}))+\widetilde{\sigma}_{3}(|\mathbf{d}|) \leqslant-\widetilde{\alpha}_{8}(U)+\widetilde{\sigma}_{3}(|\mathbf{d}|)
$$

where $\tilde{\alpha}_{8}(s)=\min \left\{\alpha_{8}(0.5 s), \kappa(\sqrt{s})\right\}$ and $\tilde{\sigma}_{3}(s)=0.5 \lambda(s)^{2}+0.5 s^{2}+\sigma_{3}(s)$. System (7) possesses UO property with respect to output $\mathbf{y}$; therefore, closed-loop system admits the same property with respect to the auxiliary output $U$. Since function $U$ admits requirements (2) and (3), then it is an IOS-Lyapunov function that according to Theorem 1 is equivalent to IOS of the system.

Substituting control (13) into estimate (A4) we again obtain (A5) that with the second part of Assumption 4 is equivalent to

$$
\begin{aligned}
\dot{U} & \leqslant-\alpha_{9}(V(\mathbf{x}))+\sigma_{4}(|\mathbf{z}-\mathbf{k}(\mathbf{x})|)+\sigma_{4}\left(\left|\mathbf{d}_{1}\right|\right)-(\mathbf{z}-\mathbf{k}(\mathbf{x}))^{\mathrm{T}} \varphi(\mathbf{z}-\mathbf{k}(\mathbf{x}))+0.5 \lambda\left(\left|\mathbf{d}_{1}\right|\right)^{2}+0.5\left|\mathbf{d}_{2}\right|^{2} \\
& \leqslant-\widetilde{\alpha}_{9}(U)+\widetilde{\sigma}_{4}(|\mathbf{d}|)
\end{aligned}
$$

where $\widetilde{\alpha}_{9}(s)=\min \left\{\alpha_{9}(0.5 s), \widetilde{\kappa}(\sqrt{s})\right\}$ and $\widetilde{\sigma}_{4}(s)=0.5 \lambda(s)^{2}+0.5 s^{2}+\sigma_{4}(s)$. In this case function $U$ again admits all requirements to be IOS-Lyapunov function and from Theorem 1 IOS property holds.

Expression (A6) for the third part of Assumption 4 can be rewritten as follows:

$$
\begin{aligned}
\dot{U} & \leqslant-\alpha_{10}(|\psi(\mathbf{x})|)+\sigma_{5}(|\mathbf{z}-\mathbf{k}(\mathbf{x})|)+\sigma_{5}\left(\left|\mathbf{d}_{1}\right|\right)-(\mathbf{z}-\mathbf{k}(\mathbf{x}))^{\mathrm{T}} \varphi(\mathbf{z}-\mathbf{k}(\mathbf{x}))+0.5 \lambda\left(\left|\mathbf{d}_{1}\right|\right)^{2}+0.5\left|\mathbf{d}_{2}\right|^{2} \\
& \leqslant-\alpha_{10}(|\psi(\mathbf{x})|)-\widetilde{\kappa}(|\mathbf{z}-\mathbf{k}(\mathbf{x})|)+\widetilde{\sigma}_{5}(|\mathbf{d}|)
\end{aligned}
$$

where $\tilde{\sigma}_{5}(s)=0.5 \lambda(s)^{2}+0.5 s^{2}+\sigma_{5}(s)$. Set $\mathscr{A}=\{(\mathbf{x}, \mathbf{z}): \mathbf{x} \in \mathscr{Z}, \mathbf{z}=\mathbf{k}(\mathbf{x})\}$ is compact due to by conditions of the theorem set $\mathscr{Z}$ is a compact one. Function $U$ is positive definite and radially unbounded with respect to set $\mathscr{A}$. In this case, the system possesses all requirements of Theorem B1 presented in Appendix B. Indeed system is uniformly globally asymptotically stable (uGAS) with respect to set $\mathscr{A}$ for zero input $\mathbf{d}$ (to prove this fact in conditions of the theorem we should apply Lemma B1) and admits estimate $\dot{U} \leqslant \widetilde{\sigma}_{5}(|\mathbf{d}|)$. The desired conclusion follows from the result of Theorem B1.

Proof of Corollary 1

Differential equation of error e dynamics for system (5), (6), (13) has the form

$$
\dot{\mathbf{e}}=-\varphi(\mathbf{e})-0.5\left(1+b(\mathbf{x}, \mathbf{z})^{2}\right) \mathbf{e}+\mathbf{d}_{2}+\frac{\partial \mathbf{k}}{\partial \mathbf{x}} \mathbf{f}(\mathbf{x}, \mathbf{z}, 0)-\frac{\partial \mathbf{k}}{\partial \mathbf{x}} \mathbf{f}\left(\mathbf{x}, \mathbf{z}, \mathbf{d}_{1}\right)
$$


This system has Lyapunov function $W(\mathbf{e})=0.5 \mathbf{e}^{\mathrm{T}} \mathbf{e}$, which time derivative can be rewritten as follows:

$$
\begin{aligned}
\dot{W} & =\mathbf{e}^{\mathrm{T}}\left(\begin{array}{r}
-\varphi(\mathbf{e})-0.5\left(1+b(\mathbf{x}, \mathbf{z})^{2}\right) \mathbf{e}+\mathbf{d}_{2} \\
+\frac{\partial \mathbf{k}}{\partial \mathbf{x}} \mathbf{f}(\mathbf{x}, \mathbf{z}, 0)-\frac{\partial \mathbf{k}}{\partial \mathbf{x}} \mathbf{f}\left(\mathbf{x}, \mathbf{z}, \mathbf{d}_{1}\right)
\end{array}\right) \\
& \leqslant-\kappa(|\mathbf{e}|)-0.5\left(1+b(\mathbf{x}, \mathbf{z})^{2}\right)|\mathbf{e}|^{2}+|\mathbf{e}|\left(\left|\mathbf{d}_{2}\right|+b(\mathbf{x}, \mathbf{z}) \lambda\left(\left|\mathbf{d}_{1}\right|\right)\right) \\
& \leqslant-\kappa(|\mathbf{e}|)-0.5\left|\mathbf{d}_{2}\right|^{2}-0.5 \lambda\left(\left|\mathbf{d}_{1}\right|\right)^{2}
\end{aligned}
$$

The last inequality implies IOS property of the system from input $\mathbf{d}$ to output e. Therefore there exist functions $\beta_{e} \in \mathscr{K} \mathscr{L}$ and $\gamma_{e} \in \mathscr{K}$ such, that for all initial conditions $\mathbf{e}_{0} \in R^{m}$ and $t \geqslant 0$ the following estimate holds:

$$
\left|\mathbf{e}\left(t, \mathbf{e}_{0}, \mathbf{d}\right)\right| \leqslant \beta_{e}\left(\left|\mathbf{e}_{0}\right|, t\right)+\gamma_{e}(\|\mathbf{d}\|)
$$

Norm of vector $\mathbf{e}_{0}$ is used as an argument of function $\beta_{e}$ due to properties of function $W$. Let the first part of conditions of the corollary be satisfied, when system (7) is UO and IOS. In this case from boundedness of variable $\mathbf{e}$ and input $\mathbf{d}$ the boundedness of output $\mathbf{y}$ follows. For UO system it implies existence of solutions $\mathbf{x}(t)$ of system (7) for all $t \geqslant 0$. Due to boundedness of error $\mathbf{e}=\mathbf{z}-\mathbf{k}(\mathbf{x})$ and continuity of control law $\mathbf{k}$ we obtain, that solution $\mathbf{z}(t)$ is defined for all $t \geqslant 0$ too. Thus, we substantiate forward completeness property of system (5), (6), (13). Property IOS of system (7) implies that there exist two functions $\beta \in \mathscr{K} \mathscr{L}$ and $\gamma \in \mathscr{K}$ such, that for all initial conditions $\mathbf{x}_{0} \in R^{n}, \mathbf{z}_{0} \in R^{m}$ and $t \geqslant 0$ the following estimate is satisfied:

$$
\left|\mathbf{y}\left(t,\left(\mathbf{x}_{0}, \mathbf{z}_{0}\right), \mathbf{d}_{1}\right)\right| \leqslant \beta\left(\left|\left(\mathbf{x}_{0}, \mathbf{z}_{0}\right)\right|, t\right)+\gamma\left(\left\|\mathbf{d}_{1}\right\|\right)+\gamma(\|\mathbf{e}\|)
$$

Combining estimates (A7), (A8) it is possible to prove boundedness of the output (y, e) for any bounded input $\mathbf{d}$ and convergence of output $\mathbf{y}$ to zero for $\mathbf{d}=0$. Let the second part of conditions of the corollary hold and system (7) be output convergent for zero input and BIBS with respect to input $\left(\mathbf{e}, \mathbf{d}_{1}\right)$. Under the same arguments the boundedness of output $\mathbf{y}$ and state $\mathbf{x}$ follows from the boundedness of variable $\mathbf{e}$ and input $\mathbf{d}$. Due to boundedness of error $\mathbf{e}=\mathbf{z}-\mathbf{k}(\mathbf{x})$ and continuity of control law $\mathbf{k}$ we obtain that solution $\mathbf{z}(t)$ is also bounded. Thus, we prove the BIBS property of system (5), (6), (13). Now assume that $\mathbf{d}(t) \equiv 0, t \geqslant 0$. Then according to (A7) variable $\mathbf{e}(t)$ asymptotically converges to zero. The convergence to zero of output $\mathbf{y}$ follows from the same property of system (7).

\section{APPENDIX B}

Consider the dynamical nonlinear system

$$
\dot{\mathbf{x}}=\mathbf{f}(\mathbf{x}, \mathbf{u})
$$

where $\mathbf{x} \in R^{n}$ is a state space vector, $\mathbf{u} \in R^{m}$ is a control or disturbing input, Lebesgue measurable and essentially bounded function of time, that is $\mathbf{u} \in \mathscr{M}_{R^{m}}$; $\mathbf{f}$ is a locally Lipschitz continuous vector field in $R^{n}, \mathbf{f}(0,0)=0$. Then $\mathbf{x}\left(t, \mathbf{x}_{0}, \mathbf{u}\right)$ denotes the unique maximal solution with the initial value $\mathbf{x}_{0} \in R^{n}$ and given $\mathbf{u} \in \mathscr{M}_{R^{m}}$ defined at least locally. It is assumed that system (B1) has a 
uGAS set $\mathscr{A}$ for $\mathbf{u} \equiv 0$ in the sense of definitions from [21]. Then according to that work the system possesses smooth Lyapunov function $V: R^{n} \rightarrow R_{+}$with respect to set $\mathscr{A}$ :

$$
\alpha_{1}\left(|\mathbf{x}|_{\mathscr{A}}\right) \leqslant V(\mathbf{x}) \leqslant \alpha_{2}\left(|\mathbf{x}|_{\mathscr{A}}\right), \quad \frac{\partial V}{\partial \mathbf{x}} \mathbf{f}(\mathbf{x}, 0) \leqslant-\alpha_{3}\left(|\mathbf{x}|_{\mathscr{A}}\right)
$$

where $\alpha_{1}, \alpha_{2}, \alpha_{3}$ are from class $\mathscr{K}_{\infty}$; the function $V$ is positive definite and radially unbounded with respect to the set $\mathscr{A}$. For compact $\mathscr{A}$ it is possible to simplify requirements for the function $V$ as follows. Before we proceed note that for compact set

$$
|\mathbf{x}| \mathscr{A} \leqslant|\mathbf{x}|+R, \quad|\mathbf{x}| \leqslant|\mathbf{x}|_{\mathscr{A}}+R
$$

where $R=\sup _{\boldsymbol{\eta} \in \mathscr{A}}|\boldsymbol{\eta}|$ is a maximal radius of set $\mathscr{A}$.

\section{Lemma B1}

System (B1) is uGAS with respect to a compact set $\mathscr{A}$ with zero input $\mathbf{u}$ if and only if there exist a smooth function $V: R^{n} \rightarrow R_{+}$and a locally Lipschitz continuous function $\psi: R^{n} \rightarrow R^{p}$ such that

$$
\begin{aligned}
\alpha_{1}\left(|\mathbf{x}|_{\mathscr{A}}\right) & \leqslant V(\mathbf{x}) \leqslant \alpha_{2}\left(|\mathbf{x}|_{\mathscr{A}}\right), \quad|\psi(\mathbf{x})| \leqslant \kappa\left(|\mathbf{x}|_{\mathscr{A}}\right) \\
\frac{\partial V}{\partial \mathbf{x}} \mathbf{f}(\mathbf{x}, 0) & \leqslant-\alpha_{3}(|\psi(\mathbf{x})|), \quad \kappa, \alpha_{1}, \alpha_{2} \in \mathscr{K}_{\infty}, \quad \alpha_{3} \in \mathscr{K}
\end{aligned}
$$

for all $\mathbf{x} \in R^{n}$ and for all $\mathbf{x}_{0} \in R^{n}$

$$
\psi\left(\mathbf{x}\left(t, \mathbf{x}_{0}, 0\right)\right) \equiv 0, \quad t \geqslant 0 \Rightarrow \lim _{t \rightarrow+\infty}\left|\mathbf{x}\left(t, \mathbf{x}_{0}, 0\right)\right|_{\mathscr{A}}=0
$$

Proof

The necessity follows from the result of [21] and (B2) with $|\psi(\mathbf{x})|=|\mathbf{x}| \mathscr{A}$. To prove sufficiency firstly note that $\dot{V} \leqslant 0$, from compactness of $\mathscr{A}$ this fact means the boundedness of $\mathbf{x}(t)$ and $\left|\mathbf{x}\left(t, \mathbf{x}_{0}, 0\right)\right|_{\mathscr{A}} \leqslant \alpha_{1}^{-1} \circ \alpha_{2}\left(\left|\mathbf{x}_{0}\right|_{\mathscr{A}}\right)$ for all $t \geqslant 0$. Applying to the system Lemma 1 we obtain that there exists some function $\beta \in \mathscr{K} \mathscr{L}$ such that

$$
\left|\psi\left(\mathbf{x}\left(t, \mathbf{x}_{0}, 0\right)\right)\right| \leqslant \beta\left(\left|\mathbf{x}_{0}\right|, t\right), \quad t \geqslant 0
$$

From the detectability property it also follows that there exists some function $\beta^{\prime} \in \mathscr{K} \mathscr{L}$ such that

$$
\left|\mathbf{x}\left(t, \mathbf{x}_{0}, 0\right)\right|_{\mathscr{A}} \leqslant \beta^{\prime}\left(\left|\mathbf{x}_{0}\right|, t\right) \leqslant \beta^{\prime}\left(\left|\mathbf{x}_{0}\right|_{\mathscr{A}}+R, t\right), \quad t \geqslant 0
$$

Then

$$
\begin{aligned}
\left|\mathbf{x}\left(t, \mathbf{x}_{0}, 0\right)\right|_{\mathscr{A}}^{2} & =\left|\mathbf{x}\left(t, \mathbf{x}_{0}, 0\right)\right|_{\mathscr{A}} \times\left|\mathbf{x}\left(t, \mathbf{x}_{0}, 0\right)\right|_{\mathscr{A}} \\
& \leqslant \beta^{\prime}\left(\left|\mathbf{x}_{0}\right|_{\mathscr{A}}+R, t\right) \times \alpha_{1}^{-1} \circ \alpha_{2}\left(\left|\mathbf{x}_{0}\right|_{\mathscr{A}}\right) \\
& \leqslant \beta^{\prime \prime}\left(\left|\mathbf{x}_{0}\right|_{\mathscr{A}}, t\right)
\end{aligned}
$$

where $\beta^{\prime \prime} \in \mathscr{K} \mathscr{L}$.

A positive-definite function $\widetilde{V}: R^{n} \rightarrow R_{+}$is called semi-proper [31], if there exist radially unbounded positive-definite function $V: R^{n} \rightarrow R_{+}$and function $\pi \in \mathscr{K}$, such that $\widetilde{V}(\mathbf{x})=\pi \circ V(\mathbf{x})$. The following result is a small development of Proposition 2.5 proposed in [31]. 


\section{Lemma B2}

System (B1) is uGAS with respect to compact set $\mathscr{A}$ with zero input $\mathbf{u}$ if and only if there exist smooth semi-proper function $\widetilde{V}: R^{n} \rightarrow R_{+}$, a function $\sigma \in \mathscr{K}$ and continuous positive-definite function $\rho: R_{+} \rightarrow R_{+}$, such that

$$
\frac{\partial \widetilde{V}}{\partial \mathbf{x}} \mathbf{f}(\mathbf{x}, \mathbf{u}) \leqslant-\rho(|\mathbf{x}| \mathscr{A})+\sigma(|\mathbf{u}|)
$$

for all $\mathbf{x} \in R^{n}$ and $\mathbf{u} \in R^{m}$.

Proof

If (B3) holds, then system (B1) is zero input uGAS for set $\mathscr{A}$ by the standard Lyapunov arguments $(\tilde{V}(\mathbf{x})=\pi \circ V(\mathbf{x})$ and $V$ is a Lyapunov function for the system with $\mathbf{u} \equiv 0)$. Conversely, if system (B1) is uGAS with respect to a compact set $\mathscr{A}$ with zero input $\mathbf{u}$, then inequalities (B2) hold for some smooth function $V$. Following [31] (proof of Proposition 2.5) there exists a function $\omega \in \mathscr{K}$ such that

$$
|\mathbf{f}(\mathbf{x}, \mathbf{u})-\mathbf{f}(0, \mathbf{u})-\mathbf{f}(\mathbf{x}, 0)| \leqslant \omega(|\mathbf{x}|) \omega(|\mathbf{u}|)
$$

then

$$
\begin{aligned}
\frac{\partial V}{\partial \mathbf{x}} \mathbf{f}(\mathbf{x}, \mathbf{u}) & \leqslant \frac{\partial V}{\partial \mathbf{x}} \mathbf{f}(\mathbf{x}, 0)+\frac{\partial V}{\partial \mathbf{x}}[\mathbf{f}(\mathbf{x}, \mathbf{u})-\mathbf{f}(0, \mathbf{u})-\mathbf{f}(\mathbf{x}, 0)]+\frac{\partial V}{\partial \mathbf{x}} \mathbf{f}(0, \mathbf{u}) \\
& \leqslant-\alpha_{3}\left(|\mathbf{x}|_{\mathscr{A}}\right)+\left|\frac{\partial V}{\partial \mathbf{x}}\right| \omega(|\mathbf{x}|) \omega(|\mathbf{u}|)+\left|\frac{\partial V}{\partial \mathbf{x}}\right||\mathbf{f}(0, \mathbf{u})|
\end{aligned}
$$

Smooth function $V$ is positive definite with respect to a set $\mathscr{A}$, and function $\partial V / \partial \mathbf{x}$ admits the same property; thus, there exists a function $\kappa \in \mathscr{K}$ which dominates $\partial V / \partial \mathbf{x}$ :

$$
|\partial V / \partial \mathbf{x}| \leqslant \kappa(|\mathbf{x}| \mathscr{A})
$$

by continuity properties of function $\mathbf{f}$ also inequality

$$
|\mathbf{f}(0, \mathbf{u})| \leqslant \mu(|\mathbf{u}|)
$$

is true for some $\mu \in \mathscr{K}$. Therefore, the following estimate

$$
\frac{\partial V}{\partial \mathbf{x}} \mathbf{f}(\mathbf{x}, \mathbf{u}) \leqslant-\alpha_{3}\left(|\mathbf{x}|_{\mathscr{A}}\right)+\kappa(|\mathbf{x}| \mathscr{A}) \omega(|\mathbf{x}|) \omega(|\mathbf{u}|)+\kappa(|\mathbf{x}| \mathscr{A}) \mu(|\mathbf{u}|)
$$

is satisfied. Then

$$
\frac{\partial V}{\partial \mathbf{x}} \mathbf{f}(\mathbf{x}, \mathbf{u}) \leqslant-\alpha_{3}\left(|\mathbf{x}|_{\mathscr{A}}\right)+\lambda(|\mathbf{x}| \mathscr{A}) \sigma(|\mathbf{u}|)
$$

where $\lambda(s)=\kappa(s)(1+\omega(2 s)+\omega(2 R))$ and $\sigma(s)=\omega(s)+\mu(s)$. Let us analyze the new semi-proper function

$$
\widetilde{V}(\mathbf{x})=\pi \circ V(\mathbf{x}), \quad \pi(r)=\int_{0}^{r} \frac{\mathrm{d} s}{1+\chi(s)}
$$


with some function $\chi \in \mathscr{K}$ to be defined later. In such a case, the time derivative of function $\widetilde{V}$ can be rewritten as follows using (B4):

$$
\begin{aligned}
\frac{\partial \widetilde{V}}{\partial \widetilde{V}} \mathbf{f}(\mathbf{x}, \mathbf{u}) & \leqslant-\frac{\alpha_{3}\left(|\mathbf{x}|_{\mathscr{A}}\right)}{1+\chi(V(\mathbf{x}))}+\frac{\lambda\left(|\mathbf{x}|_{\mathscr{A}}\right)}{1+\chi(V(\mathbf{x}))} \sigma(|\mathbf{u}|) \\
& \leqslant-\frac{\alpha_{3}\left(|\mathbf{x}|_{\mathscr{A}}\right)}{1+\chi \circ \alpha_{1}\left(|\mathbf{x}|_{\mathscr{A}}\right)}+\frac{\lambda\left(|\mathbf{x}|_{\mathscr{A}}\right)}{1+\chi \circ \alpha_{1}\left(|\mathbf{x}|_{\mathscr{A}}\right)} \sigma(|\mathbf{u}|)
\end{aligned}
$$

that for $\chi(s)=\lambda \circ \alpha_{1}^{-1}(s)$ implies desired property (B3) for $\rho(s)=\alpha_{3}(s) /(1+\lambda(s))$.

In [31] several characterizations of the iISS property [32] were introduced. Here we present a simple development of that results for the case of compact sets.

\section{Definition B1}

Forward complete system (B1) is called iISS with respect to the closed invariant set $\mathscr{A}$ if there exist functions $\alpha \in \mathscr{K}_{\infty}, \gamma \in \mathscr{K}$ and $\beta \in \mathscr{K} \mathscr{L}$ such that for any $\mathbf{x}_{0} \in R^{n}$ and all $\mathbf{u} \in \mathscr{M}_{R^{m}}$, the solution $\mathbf{x}\left(t, \mathbf{x}_{0}, \mathbf{u}\right)$ is defined for all $t \geqslant 0$ and inequality

$$
\alpha\left(\left|\mathbf{x}\left(t, \mathbf{x}_{0}, \mathbf{u}\right)\right|_{\mathscr{A}}\right) \leqslant \beta\left(\left|\mathbf{x}_{0}\right|_{\mathscr{A}}, t\right)+\int_{0}^{t} \gamma(|\mathbf{u}(\tau)|) \mathrm{d} \tau
$$

holds for all $t \geqslant 0$.

\section{Definition $B 2$}

The continuously differentiable function $W: R^{n} \rightarrow R_{+}$is called an iISS Lyapunov function with respect to a closed invariant set $\mathscr{A}$ for system (B1) if there exist functions $\alpha_{1}, \alpha_{2} \in \mathscr{K}_{\infty}, \sigma \in \mathscr{K}$ and continuous positive-definite function $\alpha_{3}$ such that

$$
\alpha_{1}\left(|\mathbf{x}|_{\mathscr{A}}\right) \leqslant W(\mathbf{x}) \leqslant \alpha_{2}\left(|\mathbf{x}|_{\mathscr{A}}\right), \quad \frac{\partial W}{\partial \mathbf{x}} \mathbf{f}(\mathbf{x}, \mathbf{u}) \leqslant-\alpha_{3}\left(|\mathbf{x}|_{\mathscr{A}}\right)+\sigma(|\mathbf{u}|)
$$

for all $\mathbf{x} \in R^{n}$ and $\mathbf{u} \in R^{m}$.

If in the above definition we impose $\alpha_{3} \in \mathscr{K}_{\infty}$, then one can recover $W$ as ISS- or IOS-Lyapunov function with respect to a set $\mathscr{A}$ from papers [13,24]. The following theorem presents only sufficient conditions for system (B1) to be iISS with respect to a compact set, while the main result in [31] provides complete equivalent characterizations for the iISS property with respect to the origin.

\section{Theorem B1}

System (B1) is iISS with respect to compact set $\mathscr{A}$ if one of the following conditions is fulfilled:

1. The system has iISS Lyapunov function with respect to set $\mathscr{A}$.

2. Set $\mathscr{A}$ is uGAS for the system with zero input $\mathbf{u}$ and for system (B1) there exists continuously differentiable, positive definite and radially unbounded with respect to set $\mathscr{A}$ function $U$ : $R^{n} \rightarrow R_{+}$with

$$
\frac{\partial U}{\partial \mathbf{x}} \mathbf{f}(\mathbf{x}, \mathbf{u}) \leqslant v(|\mathbf{u}|), \quad v \in \mathscr{K}
$$

for all $\mathbf{x} \in R^{n}$ and $\mathbf{u} \in R^{m}$. 
3. There exist continuously differentiable function $V: R^{n} \rightarrow R_{+}$and continuous function $\psi$ : $R^{n} \rightarrow R^{p}$ such that

$$
\begin{aligned}
\alpha_{1}\left(|\mathbf{x}|_{\mathscr{A}}\right) & \leqslant V(\mathbf{x}) \leqslant \alpha_{2}\left(|\mathbf{x}|_{\mathscr{A}}\right), \quad|\psi(\mathbf{x})| \leqslant \kappa\left(|\mathbf{x}|_{\mathscr{A}}\right) \\
\frac{\partial V}{\partial \mathbf{x}} \mathbf{f}(\mathbf{x}, \mathbf{u}) & \leqslant-\alpha_{3}(|\psi(\mathbf{x})|)+\lambda(|\mathbf{u}|), \quad \kappa, \lambda, \alpha_{1}, \alpha_{2}, \alpha_{3} \in \mathscr{K}_{\infty}
\end{aligned}
$$

for all $\mathbf{x} \in R^{n}, \mathbf{u} \in R^{m}$ and for all $\mathbf{x}_{0} \in R^{n}$

$$
\psi\left(\mathbf{x}\left(t, \mathbf{x}_{0}, 0\right)\right) \equiv 0, \quad t \geqslant 0 \Rightarrow \lim _{t \rightarrow+\infty}\left|\mathbf{x}\left(t, \mathbf{x}_{0}, 0\right)\right|_{\mathscr{A}}=0
$$

Proof

To prove that the first condition of the theorem implies iISS with respect to set $\mathscr{A}$ it is possible to simply apply Corollary 4.3 from [31]. According to the second condition it is possible to note that all conditions of Lemma B2 are satisfied and semi-proper function $\widetilde{V}: R^{n} \rightarrow R_{+}$exists such that for some function $\sigma \in \mathscr{K}$ and continuous positive- definite function $\rho: R_{+} \rightarrow R_{+}$inequality (B3) holds for all $\mathbf{x} \in R^{n}$ and $\mathbf{u} \in R^{m}$. Let us consider function $W(\mathbf{x})=\widetilde{V}(\mathbf{x})+U(\mathbf{x})$ as an iISS Lyapunov function candidate. It is clear that this function admits all conditions of Definition B2. Combining the third condition and the result of Lemma B1 we obtain that the third condition implies the second one.

\section{ACKNOWLEDGEMENTS}

This work is partly supported by grant 05-01-00869 of Russian Foundation for Basic Research and by Program of Presidium of Russian Academy of Science No. 22.

\section{REFERENCES}

1. Kokotović PV, Arcak M. Constructive nonlinear control: a historical perspective. Automatica 2001; 37:637-662.

2. Artstein Z. Stabilization with relaxed control. Nonlinear Analysis 1983; 7:1163-1173.

3. Efimov DV. A condition of CLF existence for affine systems. Proceedings of the 41st IEEE Conference on Decision and Control, Las Vegas, 2002.

4. Sontag ED. A 'universal' construction of Arstein's theorem on nonlinear stabilization. Systems and Control Letters 1989; 12:542-550.

5. Isidori A. Nonlinear Control Systems: An Introduction (2nd edn). Springer: Berlin, 1989; 478.

6. Seron M, Hill DJ, Fradkov AL. Nonlinear adaptive control of feedback passive systems. Automatica 1995; 31:1053-1060.

7. Fradkov AL, Miroshnik IV, Nikiforov VO. Nonlinear and Adaptive Control of Complex Systems. Kluwer: Dordrecht, 1999; 528.

8. Kolesnikov AA. Synergetic Control Theory. Energoatomizdat: Moscow, 1994; 344 (in Russian).

9. Krstić M, Kanellakopoulos I, Kokotović PV. Nonlinear and Adaptive Control Design. Wiley: New York, 1995; 563.

10. Sepulchre R, Jankovic M, Kokotović P. Constructive Nonlinear Control. Springer: New York, 1997.

11. Teel AR. Using saturation to stabilize a class of single-input partially linear composite systems. In Preparation of the 2nd IFAC Nonlinear Control Systems Design Symposium, Bordeaux, France, 1992; 224-229.

12. Sontag ED. Smooth stabilization implies coprime factorization. IEEE Transactions on Automatic Control 1989; 34:435-443.

13. Sontag ED, Wang Y. Various results concerning set input-to-state stability. Proceedings of IEEE CDC 95. IEEE Publications: New York, 1995; 1330-1335. 
14. Sontag ED, Wang Y. On characterization of the input to state stability property. Systems and Control Letters 1995; 24:351-359.

15. Rumyantsev VV, Oziraner AS. Stability and Stabilization of Motion with Respect to Part of Variables. Nauka: Moscow, 1987; 263 (in Russian).

16. Shiriaev AS, Canudas-de-Wit C. Virtual constraints: a tool for orbital stabilization of nonlinear systems theory. Proceedings of the 6th IFAC Symposium NOLCOS 2004, Stuttgart, 2004; 1355-1360.

17. Shiriaev AS, Fradkov AL. Stabilization of invariant sets for nonlinear non-affine systems. Automatica 2000; 36:1709-1715.

18. Shiriaev AS, Fradkov AL. Stabilization of invariant sets for nonlinear systems with application to control of oscillations. International Journal of Robust and Nonlinear Control 2001; 11:215-240.

19. Skjetne R, Teel AR, Kokotović PV. Nonlinear maneuvering with gradient optimization. Proceedings of CDC 2001, Orlando, FL, U.S.A., 2001; 3926-3931. Paper FrA11-1.

20. Vorotnikov VI. Partial Stability and Control. Birkhauser: Boston, MA, 1998; 442.

21. Lin Y, Sontag ED, Wang Y. A smooth converse Lyapunov theorem for robust stability. SIAM Journal on Control and Optimization 1996; 34:124-160.

22. Ingalls B, Wang Y. On input-to-output stability for systems not uniformly bounded. Proceedings of NOLCOS'01, Saint-Petersburg, Russia, 2001.

23. Sontag ED, Wang Y. Notions of input to output stability. Systems and Control Letters 1999; 38:235-248.

24. Sontag ED, Wang Y. Lyapunov characterizations of input to output stability. SIAM Journal on Control and Optimization 2001; 39:226-249.

25. Efimov DV. Universal formula for output asymptotic stabilization. Proceedings of the 15th IFAC World Congress 2002, Barcelona, Spain, 2002. T-We-M 074.

26. Martinez S, Cortes J, Bullo F. Analysis and design of oscillatory control systems. IEEE Transactions on Automatic Control 2003; 48(7):1164-1177.

27. Polushin IG, Fradkov AL, Putov VV, Rogov KA. Energy control of one-degree-of-freedom oscillators in presence of bounded force disturbance. Proceedings of ECC99, Karlsruhe, Germany, 1999; 6. Paper F1017-3.

28. Angeli D, Sontag ED. Forward completeness, unboundedness observability, and their Lyapunov characterizations. Systems and Control Letters 1999; 38:209-217.

29. Jiang Z-P, Teel A, Praly L. Small-gain theorem for ISS systems and applications. Mathematics of Control, Signals, and Systems 1994; 7:95-120.

30. Arcak M, Seron M, Braslavsky J, Kokotović P. Robustification of backstepping against input unmodeled dynamics. IEEE Transactions on Automatic Control 2000; 45:1-6.

31. Angeli D, Sontag ED, Wang Y. A characterization of integral input to state stability. IEEE Transactions on Automatic Control 2000; 45:1082-1097.

32. Sontag ED. Comments on integral variants of ISS. Systems and Control Letters 1998; 34:93-100. 